\title{
Nuevos reportes de ubicación y calidad de agua en los humedales altoandinos del páramo del Duende, Chocó, Colombia
}

\section{New reports of location and water quality in wetlands high Andean of the Páramo del Duende, Chocó, Colombia}

\section{Lady Vargas Porras*}

\section{RESUMEN}

El Páramo del Duende en jurisdicción del departamento de Chocó, es un ecosistema poco conocido que abarca muchos componentes de estudio dentro de los cuales se encuentra, además de una gran riqueza biológica, un componente físico que incluye el agua como el elemento más importante para su constitución, pues determina la presencia y ausencia de los demás. Con el objeto de identificar, georeferenciary analizar el estado actual de los humedales de este ecosistema se realizó un trabajo de campo que permitió el análisis de la estructura, calidad y composición de varias fuentes lénticas durante la época seca (diciembre). Para la determinación de la estructura y ubicación se realizaron recorridos a nivel de bordes con un GPS programado para la toma de coordenadas cada cinco segundos y para el análisis de composición y calidad se realizaron mediciones in situ de oxígeno disuelto, temperatura, $p H$, conductividad y profundidad media. Los resultados obtenidos se analizaron y compararon con estándares de calidad de agua para la preservación de la biota acuática. Se logró aportar al conocimiento no solo del ecosistema de páramo, sino también al análisis de la dinámica de sus humedales, además se obtuvo el reporte y la inclusión de estas fuentes en la cartografía de la zona.

Palabras clave: Páramo del Duende; Humedales altoandinos; Calidad de agua en humedales.

\begin{abstract}
The Páramo del Duende in the jurisdiction of the department of Chocó, it is an ecosystem little acquaintance who includes many components of study inside which he is besides a great biological wealth, a physical component that includes the water as the most important element for his constitution, since it determines the presence and absence of the others. In order to identify, geographically reference and to analyze the current condition of the wetlands of this ecosystem there was realized a fieldwork that allowed the analysis of the structure, quality and composition of several lentic sources during the dry epoch (December). For the determination of the structure and location tours were realized to level of edges by a GPS programmed for the capture of coordinates every 5 seconds, and for the analysis of composition and quality measurements were realized in situ of dissolved oxygen, temperature, $p H$, conductivity and depth happens. The obtained results were analyzed and compared with standards of water quality for the preservation of the aquatic biota. It was achieved to reach to the not alone knowledge of the ecosystem of high plateau, but also to the analysis of the dynamics of his wetlands, in addition there was obtained the report and the incorporation of these sources in the cartography of the zone.
\end{abstract}

* Ingeniera Ambiental y Sanitaria, Esp. Manejo Integrado de Recursos Hídricos. Contratista Instituto de Investigciones Ambientales del Pacífico (IIAP), Quibdó, Colombia. e-mail: yulenis81@yahoo.es Recibido: 16 de julio de 2011

Aceptado: 26 de octubre de 2011 
Keywords: Páramo del Duende; Wetlands high Andean; Water quality in wetlands.

\section{INTRODUCCIÓN}

El Páramo del Duende comprende el conjunto de ecosistemas de la cordillera Occidental entre el noroeste del departamento del Valle del Cauca y el sureste del departamento de Chocó. Cabe mencionar que la zona de este ecosistema correspondiente al primero, que abarca los municipios de Riofrío, Trujillo y Calima; fue declarada Parque Natural Regional en agosto de 2005; en ella se han realizado diversos estudios de tipo florístico y faunístico, además de la formulación de un plan de manejo por FEDENA et al. (2004) que incluyó delimitación, zonificación y definición de objetivos de conservación. Estos estudios han permitido contar con información tanto física como biológica y social de esa zona del Páramo del Duende, situación que no ocurre en la jurisdicción del departamento del Chocó, donde no se reportan estudios realizados que permitan determinar el estado actual del ecosistema en mención.

Como resultado de una revisión bibliográfica de toda la información existente relacionada con el componente hidrológico de esta zona, incluyendo la determinación de la estructura y composición de la red hídrica, y el análisis de parámetros fisicoquímicos en la misma, se encontró que además de existir un gran vacío de información sobre estudios de este tipo por falta de investigaciones realizadas directamente en el área correspondiente al departamento del Chocó, la información existente para el área de páramo concerniente al departamento del Valle y otros ecosistemas paramunos de la cordillera Occidental está relacionada sobre todo con la distribución de la red hídrica y en muy pocos casos con la calidad del recurso y los humedales.

El Parque Regional Natural Páramo del Duende corresponde a un área de páramo en excelente estado de conservación localizada sobre la cordillera Occidental, en jurisdicción de los municipios Riofrío y Darién, en el departamento del Valle del Cauca, y Docordó, en el Chocó. A pesar de ser menos húmedo que otros páramos localizados sobre la cordillera Occidental, allí nacen múltiples cuerpos de agua, como los ríos Calima, Bravo, Azul y Frío, entre otros citados por la organización BirdLife International (2009). El IDEAM (2006), informa que en el complejo del Duende se origina la cuenca del río San Juan y Munguidó, y la cuenca del río Calima, que confluye hacia el río San Juan del Chocó. Por esta razón, se considera importante en la regulación de aguas del Chocó biogeográfico.

En los informes de Salgado (2006) se identifican dos grandes cuencas nutridas por el Páramo del Duende, la cuenca del río San Juan (Pacífico) que recoge las aguas de los ríos San Quinini, la Playa, Copomá, Paradó, Munguidó,
Pata Sola, los Espíritus, Azul, el Militar, el Tambor, Bravo y Calima; En general, esta área presenta la gran mayoría de las corrientes de agua medianas y pequeñas sin nombre, aparentemente por la baja densidad de población ubicada en ella, y la Cuenca del río Cauca (Atlántico) que recoge las aguas que vierten en la parte centro norte del Parque Natural Regional del Duende en el municipio de Riofrío, cuyo río en un recorrido de $49 \mathrm{~km}$ recibe las aguas de los ríos Blanco, el Duende, Volcanes, Claro, Lindo, Arauca, Cristales, Medio Pañuelo, Cáceres, Chiquito, Culebras y Cuancua. Estos a su vez reciben las aguas de numerosas quebradas que a su paso surten acueductos veredales y municipales.

En cuanto a la calidad del agua ofertada por el Páramo del Duende, no se encontraron reportes de análisis pero estudios como el Plan de Manejo del Parque Natural Regional Páramo del Duende y su zona amortiguadora en la zona del Valle, indican el uso de las aguas de sus cuencas en la generación de energía de las microcentrales hidroeléctricas Riofrío I y Riofrío II y principalmente para riego agrícola de las 3597 ha en monocultivos de caña de azúcar en la zona plana de los municipios de Riofrío y Trujillo (FEDENA, et al. 2004).

En términos generales, solo se encontraron inventarios de nacimientos de ríos y quebradas de la zona, lo que permite corroborar la importancia de la oferta hídrica del páramo y la necesidad de evaluar su estado, y más aún, en lo relacionado con fuentes lénticas como humedales, ciénagas o turberas, de las cuales no se encontraron reportes ni en documentos ni cartografía, lo que resalta la urgencia de realizar estudios que permitan identificar este tipo de fuentes, que cuentan con una importancia declarada en convenios y políticas nacionales e internacionales. En este contexto y teniendo en cuenta la importancia que reviste esta clase de ecosistemas en la dinámica hídrica tanto regional como nacional, es prioritario realizar estudios y caracterizaciones orientadas al conocimiento del estado del Páramo con miras a su preservación mediante diferentes alternativas de manejo.

\section{ÁREA DE ESTUDIO}

El Páramo del Duende se localiza en la cordillera Occidental y es compartido entre los departamentos de Valle del Cauca y Chocó, se ubica por encima de los $3450 \mathrm{msnm}$ y bajo los $3830 \mathrm{msnm}$, y abarca aproximadamente 2500 ha, de las cuales 1562 ha se localizan en el departamento del Chocó jurisdicción del municipio del Litoral del San Juan que tiene como cabecera a Docordó y 316,17 hectáreas en el departamento del Valle del Cauca (Salgado 2006).

La temperatura oscila entre $\operatorname{los} 5^{\circ} \mathrm{C}$ y $13^{\circ} \mathrm{C}$. La humedad relativa con frecuencia es muy alta, entre $80 \%$ y $98 \%$ durante gran parte de la noche y el día; posee un carácter variable y estacional (máxima en época de lluvias y mínima en las estaciones secas), y además se suele presentar el fenómeno 


\section{Bioetnia Volumen 8 No 2 (julio-diciembre), 2011}

de niebla. En términos generales, el complejo se caracteriza por un paisaje altamente montañoso, de laderas disectadas, pendientes muy pronunciadas donde conforme aumenta la altitud se presentan áreas de intensa neblina y una superficie con un relieve ondulado de pendientes suaves y pequeños valles (FEDENA et al. 2004). Además, se presentan unos $7500 \mathrm{~mm}$ a $7550 \mathrm{~mm}$ anuales de precipitación, que va disminuyendo sistemáticamente hasta alcanzar valores cercanos a $5000 \mathrm{~mm}$ muy cerca de las estribaciones occidentales de la cordillera Occidental.

Las condiciones climatológicas, la ubicación, la topografía y la vegetación del páramo del Duende lo convierten en un ecosistema altamente productor de agua, donde nacen muchas fuentes hídricas de diferentes tipos, con diversos usos y rangos de importancia, como ríos, quebradas y humedales. Aunque la porción que corresponde al departamento del Chocó es muy amplia, para la presente investigación se escogieron solo los humedales que se identificaron en el área seleccionada para el muestreo.

El área de estudio específica está compuesta por fuentes lénticas en las cuales se realizaron observaciones en campo para analizar la dinámica hídrica, mediciones in situ de parámetros fisicoquímicos para hacer inferencias sobre la calidad del recurso. Los cuerpos de agua seleccionados son el complejo de humedales denominado por el equipo técnico como Citará y el humedal Luz del Carmen.

\section{METODOLOGÍA}

\section{Muestreo}

Ubicación y estructura. Se realizaron recorridos por el área para identificar los humedales, se utilizó un GPS que permitió su georeferenciación y la ubicación de los puntos de muestreo. Para determinar la estructura de las fuentes se realizaron recorridos por sus bordes con el equipo programado para la toma de coordenadas cada cinco segundos, lo que permitió obtener un área aproximada que se incluyó dentro de cartografía, teniendo en cuenta que este tipo de fuentes no se encontraban registradas en los mapas del área.

Composición y calidad. Se realizaron mediciones in situ durante el mes de diciembre que corresponde a la época seca de la zona. Los parámetros analizados fueron oxígeno disuelto, temperatura, $\mathrm{pH}$, conductividad y profundidad en diferentes puntos de acuerdo con el tamaño y la forma del humedal, para obtener resultados de la variación espacial de las condiciones del recurso, y entender la dinámica del ecosistema y el flujo de los nutrientes (Figura 1).

Los resultados obtenidos por cada parámetro analizado en los humedales se compararon con lo estipulado en el Decreto 1594 de 1984 sobre cuerpos de agua y con estándares internacionales de calidad de agua para la preservación de la biota acuática.

\section{RESULTADOS Y DISCUSIÓN}

Se lograron identificar dos humedales, el primero fue denominado por el equipo técnico como el complejo de humedales Citará y el segundo como humedal Luz del Carmen. Las características de estos se describen a continuación:

Complejo de humedales Citará. Se localiza a 3561 msnm en las coordenadas 44'19.0.39' ' Ny 76³0'30.2' 'W. Se logró deducir por el estado del terreno que en épocas de lluvia está conformado por un solo cuerpo de agua; no obstante, durante los recorridos realizados (época seca), se encontró constituido por tres espejos de agua de poca profundidad, en los que se obtuvieron las siguientes características fisicoquímicas en el agua (Tablas 1, 2 y 3 ).

La concentración de oxígeno varió de 1.15 hasta 2.29 $\mathrm{mg} / \mathrm{l}$ en los espejos de mayor tamaño y mayor profundidad; estos datos y las observaciones realizadas podrían indicar niveles de eutroficación, por el excesivo crecimiento de algas
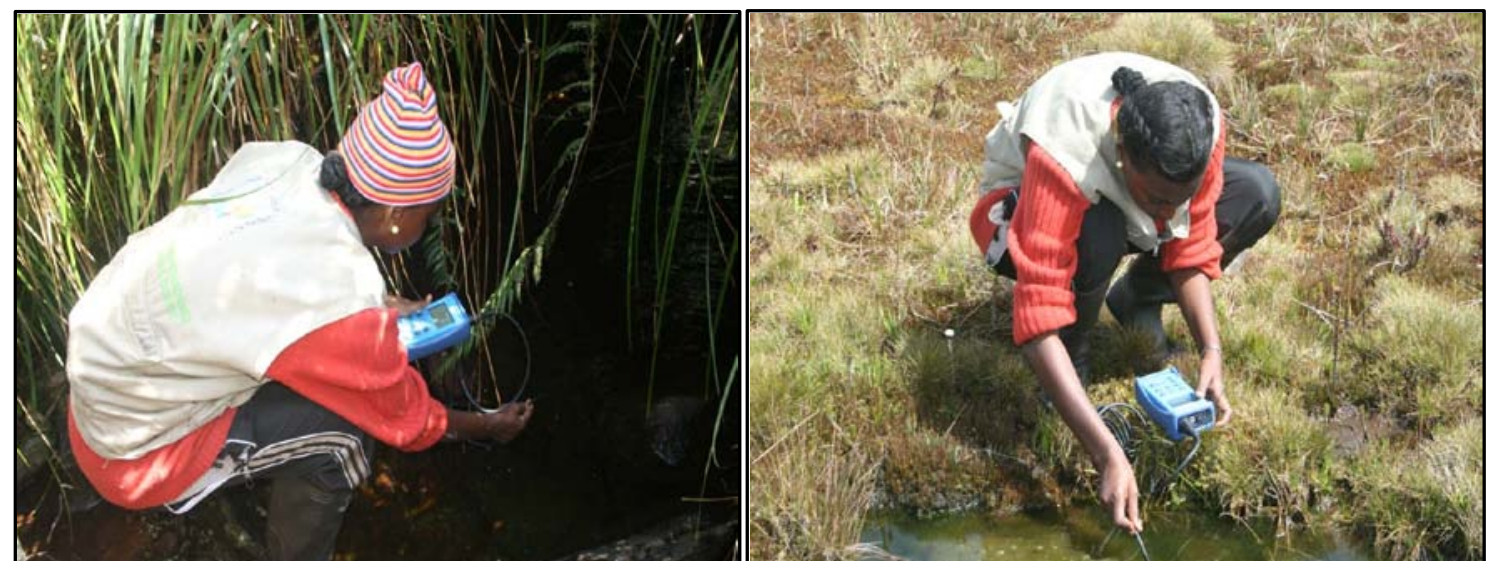

Figura 1. Medición de parámetros fisicoquímicos 
Tabla 1

Características fisicoquímicas del agua del complejo de humedales Citará. Espejo 1

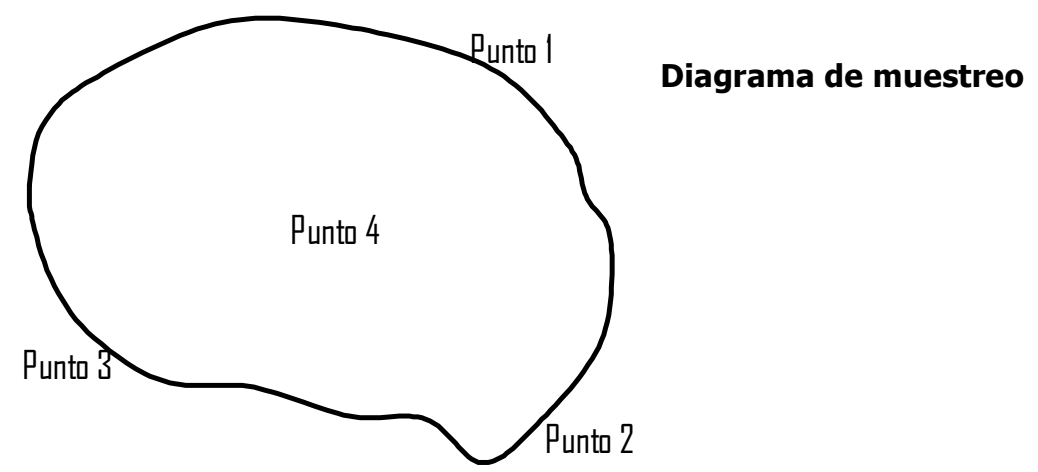

\begin{tabular}{lccccc}
\hline $\begin{array}{l}\text { Parámetro } \\
\text { Punto de } \\
\text { muestreo }\end{array}$ & $\begin{array}{c}\text { Oxigeno disuelto } \\
(\mathbf{m g} / \mathbf{l})\end{array}$ & Ph & $\begin{array}{c}\text { Conductividad } \\
(\mu \mathbf{s} / \mathbf{c m})\end{array}$ & $\begin{array}{c}\text { Temperatura } \\
\left({ }^{\circ} \mathbf{C}\right)\end{array}$ & $\begin{array}{c}\text { Profundidad media } \\
(\mathbf{c m})\end{array}$ \\
\hline Punto 1 & 1.62 & 5.01 & 10.9 & 15.0 & \\
\hline Punto 2 & 1.49 & 5.03 & 11.5 & 15.7 & \\
Punto 3 & 1.87 & 4.79 & 12.0 & 15.6 & 41.5 \\
\hline Punto 4 (Centro) & 2.29 & 4.98 & 12.0 & 15.7 & \\
\hline
\end{tabular}

Tabla 2

Características fisicoquímicas del agua del complejo de humedales Citará. Espejo 2

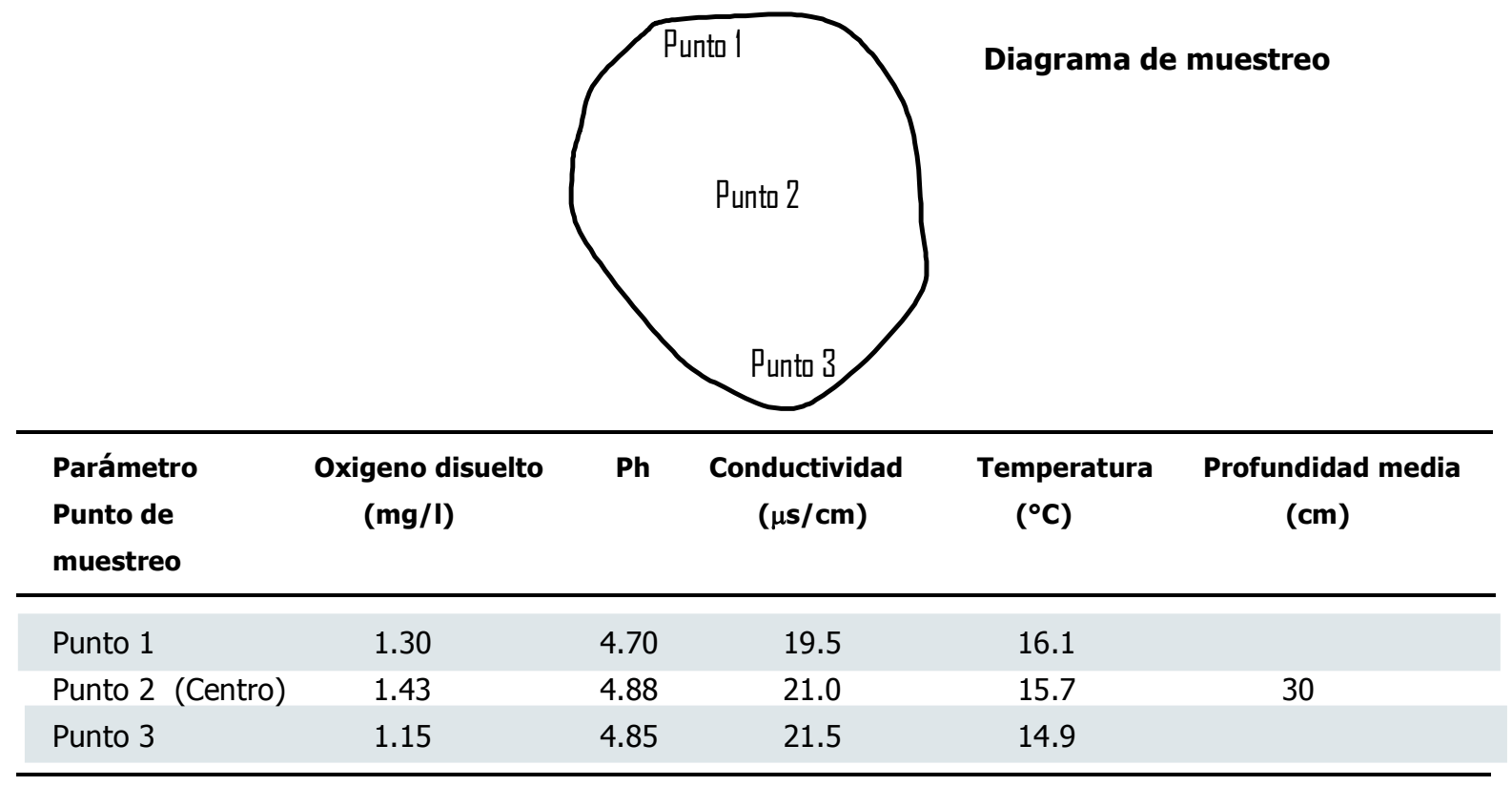


Tabla 3

Características fisicoquímicas del agua del complejo de humedales Citará. Espejo 3

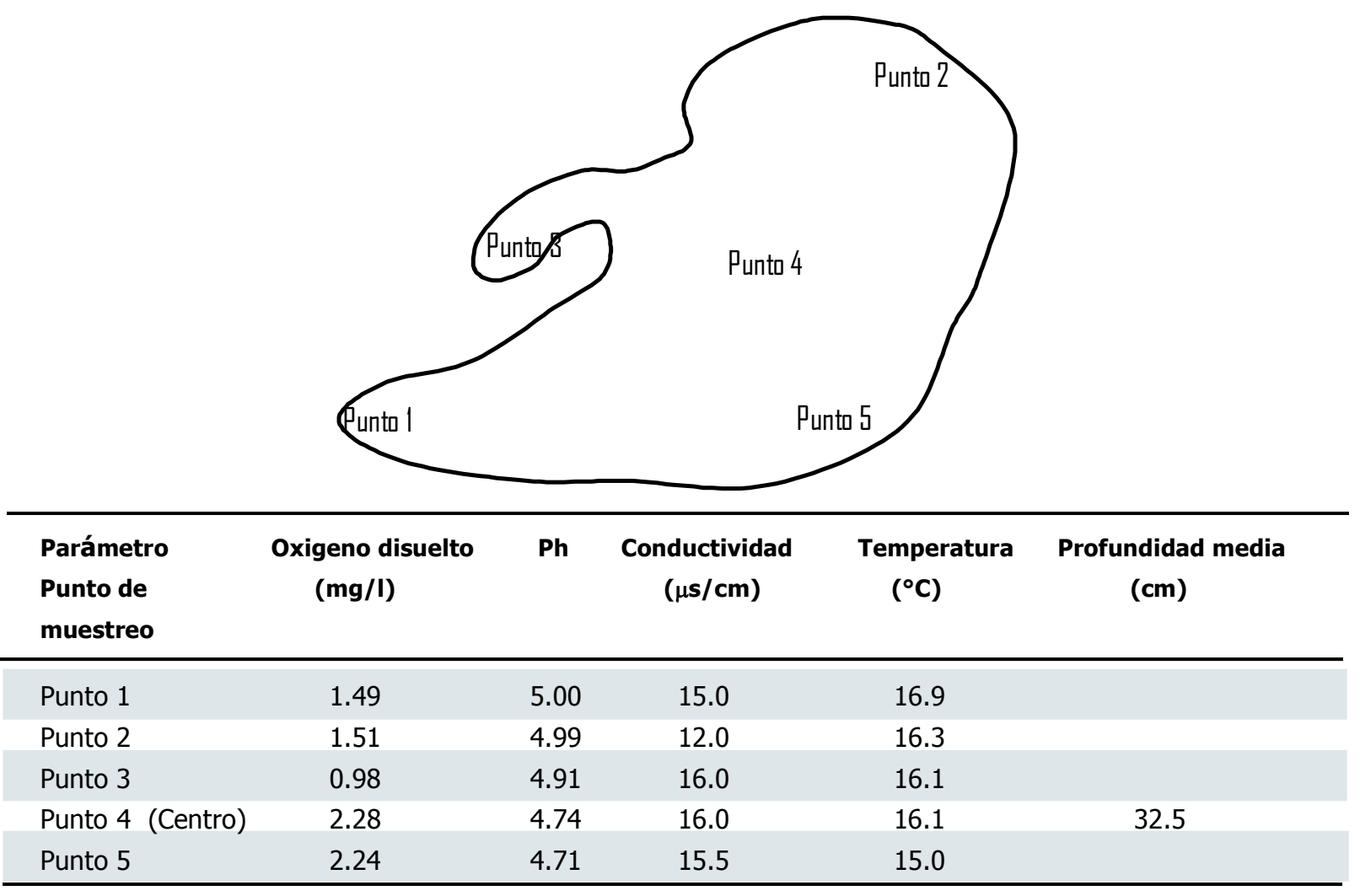

en el agua de pocas profundidades (entre $30 \mathrm{~cm} \mathrm{y} 41.5 \mathrm{~cm}$ ) y la baja disponibilidad de oxígeno. Las mediciones muestran bajos niveles de oxígeno disuelto en el agua, si se tiene en cuenta que el límite admisible para preservación de flora y fauna es de $5 \mathrm{mg} / \mathrm{l}$ según el Decreto 1594/84. Se encontró que la mayor concentración de este elemento se presenta hacia la zona central de los espejos, posiblemente por su mayor profundidad y a la saturación de algas que se observa en los bordes consumiendo la cantidad de oxígeno disponible en el agua (Figura 2).

El pH osciló entre 4.71 y 5.01 unidades, predominando siempre la condición ácida lo que podría corroborar una elevada concentración de material disuelto, resultado muy común en aguas de tipo léntico. En cuanto a la conductividad se registraron valores normales para aguas naturales, que oscilaron entre 12 hasta $21.5 \mathrm{is} / \mathrm{cm}$ en promedio, indicando presencia de materiales disueltos. Las temperaturas variaron entre $14.9^{\circ} \mathrm{C}$ hasta $16.9^{\circ} \mathrm{C}$, principalmente por la excesiva radiación solar presentada durante las horas de medición (10:00 am) y a los procesos químicos que se desarrollan al interior de los cuerpos de agua que se encargan de liberar calor.

La topografía del terreno y la vegetación resultan ser con seguridad factores determinantes en los resultados obtenidos y en la formación del humedal, porque el complejo se encuentra localizado en la parte baja de la zona montañosa, es decir, en una depresión que recibe por escorrentía gran cantidad de agua que arrastra los nutrientes y materiales que encuentra adheridos al suelo; de ahí los niveles de conductividad encontrados, las aguas ácidas y los bajos niveles de oxgeno disuelto que indicaron la presencia de materia orgánica disuelta.

Humedal Luz del Carmen. Se localiza a $3427 \mathrm{msnm}$ en las coordenadas 44'25.4' N y 76³0'49.2' 'W. Aunque se encontró un pequeño espejo de agua durante los recorridos de campo, se observó una gran área de influencia del humedal caracterizada por un terreno fangoso con gran contenido de agua, lo que indicó que en la época lluviosa presenta un gran tamaño, el cual se redujo al igual que su profundidad por el estiaje de la época de muestreo. En dicho cuerpo de agua se obtuvieron los datos fisicoquímicos que se muestran en la Tabla 4. Este humedal presenta condiciones muy similares a las que se encontraron en el complejo Citará, con bajas concentraciones de oxígeno disuelto $(1.77 \mathrm{mg} / \mathrm{l})$, temperatura de $14^{\circ} \mathrm{C}$, baja profundidad y aguas tendientes a las acidez (Figura 3). 
Tabla 4

Características fisicoquímicas del agua del humedal Luz del Carmen

\begin{tabular}{lccccc}
\hline $\begin{array}{l}\text { Parámetro } \\
\text { Punto de } \\
\text { muestreo }\end{array}$ & $\begin{array}{c}\text { Oxigeno disuelto } \\
(\mathbf{m g} / \mathbf{l})\end{array}$ & Ph & $\begin{array}{c}\text { Conductividad } \\
(\mu \mathbf{s} / \mathbf{c m})\end{array}$ & $\begin{array}{c}\text { Temperatura } \\
\left({ }^{\circ} \mathbf{C}\right)\end{array}$ & $\begin{array}{c}\text { Profundidad media } \\
(\mathbf{c m})\end{array}$ \\
\hline Punto 1 & 1.77 & 5.7 & 8.00 & 14.1 & 20 \\
\hline
\end{tabular}

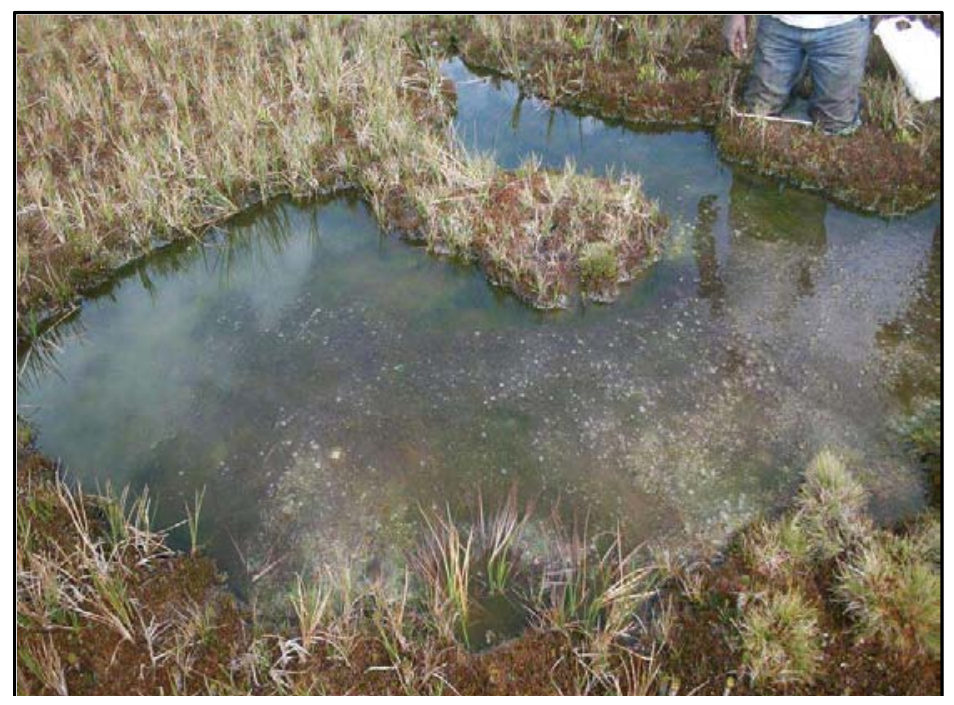

Figura 2. Espejos de agua del complejo de humedales Citará

En general, los humedales de este ecosistema de alta montaña presentan una composición de aguas ácidas de temperatura media, originada por la alta radiación que se produce durante el día y poco oxígeno disuelto cuya dinámica, formación y composición está relacionada directamente con el clima, la topografía y la vegetación, porque se forman en depresiones del terreno donde dejan de aparecer especies vegetales retenedoras de gran cantidad de agua como las bromelias, permitiendo que el recurso que precipita por acción de la escorrentía se almacene en dichas depresiones y forme ecosistemas de humedales característicos que se comportan de manera similar a los humedales de las zonas bajas pero con una dinámica de flujo de nutrientes diferente, porque en estos últimos este proceso depende por lo general de la interrelación con ríos o quebradas a través de caños visibles o subterráneos. Para el complejo cenagoso la Grande de Beté en el Medio Atrato, Chocó, se encontró que el pH es en general ácido para todas las zonas, presentándose aguas con $\mathrm{pH}$ más bajos en las zonas de rivera y de espejo de agua de la ciénaga, y valores cercanos a $\mathrm{pH}$ neutro en las zonas de caño y de influencia del río, lo que explica que el flujo de agua se comporta en dirección río-ciénaga, llevando con él gran cantidad de sólidos suspendidos y material disuelto que aunque alcanzan a llegar en menores proporciones a la ciénaga, por la filtración del suelo y la vegetación, ingresa una cantidad considerable que al iniciar su proceso de descomposición al interior del espejo genera una variación del pH del agua (Vargas 2008), situación que no ocurre en los humedales de alta montaña encontrados en el Páramo del Duende, que no presentaron caños visibles o conexiones a ríos que aporten a ellos un flujo de nutrientes, porque estos últimos presentan muy bajas concentraciones de los mismos por sus altos niveles de calidad y por la inexistencia de actividades humanas en la zona. Es decir, que estos dos tipos de humedales presentan diferencias en formación u origen, así como en su dinámica, aunque puedan mostrar composiciones similares. En este caso, para el humedal Luz del Carmen y el complejo Citará, el material disuelto lo aportan la escorrentía del agua en el proceso de formación y la vegetación circundante,y la dinámica depende de la época climática y de la topografía del terreno.

Cabe mencionar que se requiere la realización de mediciones durante otras épocas climáticas para entender el comportamiento completo de estos humedales de alta mon- 


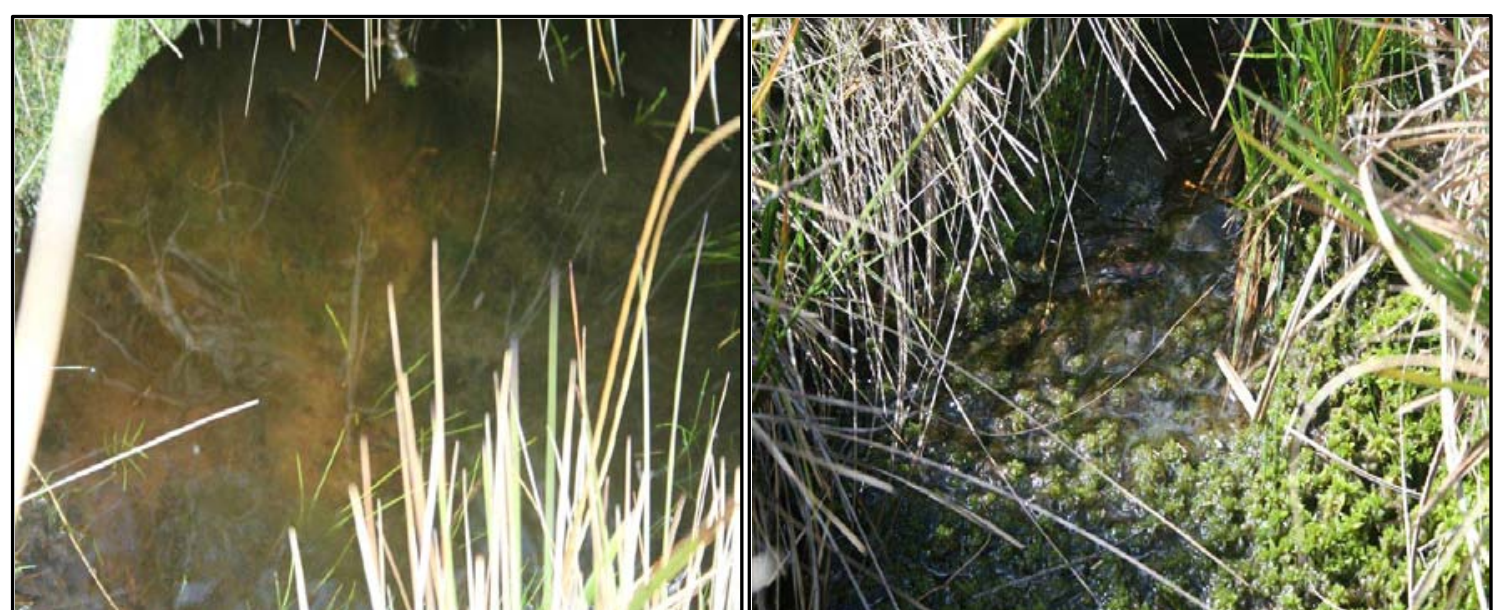

Figura 3. Espejo de agua del humedal Luz del Carmen

Tabla 5

\section{Comparación del pH de los humedales del Páramo del Duende con estándares internacionales de calidad de agua para la preservación de la biota acuática}

\begin{tabular}{|c|c|c|c|c|c|c|}
\hline \multirow[b]{2}{*}{$\begin{array}{l}\text { Parámetro } \\
\text { comparable }\end{array}$} & \multicolumn{4}{|c|}{ Estándares y Norma } & \multicolumn{2}{|c|}{ Resultados en Campo } \\
\hline & $\begin{array}{c}\text { Panamá } \\
\text { (Cooke, et al. 2001) }\end{array}$ & $\begin{array}{c}\text { Estándar } \\
\text { Argentina } \\
(\text { Carrizo, 2008) }\end{array}$ & $\begin{array}{c}\text { Uruguay } \\
\text { (MVOMAU, 2007) }\end{array}$ & $\begin{array}{c}\text { Decreto } 1594 \\
\text { /84 usos Colombia }\end{array}$ & $\begin{array}{l}\text { Complejo Citará } \\
\text { (promedio) }\end{array}$ & $\begin{array}{c}\text { Humedal } \\
\text { Luz del Carmen }\end{array}$ \\
\hline $\mathrm{pH}$ & De 5-9 & De 6.5 a 8.5 & De 6 a 9.0 & De 6.5-9.0 & 4.9 & 5.7 \\
\hline
\end{tabular}

taña, porque no se encontraron especies ícticas, lo que además del clima está ocasionado por las pocas profundidades encontradas en la época de análisis (seca), así que es probable que todas las condiciones que se mencionan varíen de forma considerable.

Si se comparan las concentraciones de $\mathrm{pH}$ obtenidas con estándares de calidad de agua para la preservación de la biota acuática, se obtienen los resultados mostrados en la Tabla 5.

Los valores de $\mathrm{pH}$ obtenidos se encuentran por lo general por fuera de los rangos establecidos por la mayoría de los estándares de calidad de preservación de la vida acuática a excepción de la Norma de Panamá, lo que muestra una particularidad del ecosistema y las especies que se desarrollan en él, porque a pesar de estos resultados, se encontró una gran variedad de especies de macroinvertebrados y algas en estos cuerpos de agua. Resultados similares de $\mathrm{pH}$ por fuera de estos rangos han sido informados en otros humedales ubicados en zonas más bajas como los que se encuentran en la meseta de Popayán y Puracé en el departamento del Cauca (Estela, et al. 2006), donde se obtuvieron para el humedal La Olímpica valores de $\mathrm{pH}$ de 5.85; este humedal se encuentra ubicado en una zona contaminada donde el valor como hábitat para el mantenimiento de la biodiversidad local es limitado por su amplia transformación en ambientes urbanos y rurales; de ahí que sus características bióticas corresponden a ambientes muy perturbados, a diferencia de la zona de estudio que presenta alto grado de conservación y poca acción antrópica, lo que hace importante el estudio de las especies que se desarrollan en estas condiciones y sus comportamientos, además de la composición del agua en otras época del año, porque las lluvias son un factor determinante en la dilución de las sustancias que contribuyen a la acidificación del agua y las concentraciones bajas de $\mathrm{pH}$.

\section{CONCLUSIONES Y ASPECTOS RELEVANTES}

La dinámica hídrica en el Páramo del Duende está directamente determinada por diversas variables como la topografía del terreno, la geología de la zona, el clima y la interrelación con la vegetación predominante y la distribución espacial de la misma, de ahí que los cuerpos de agua estén localizados de acuerdo con el comportamiento combinado de dichas variables.

Para el conocimiento amplio del comportamiento del componente agua dentro del ecosistema de Páramo del Duende y su interrelación con los componentes bióticos del mismo, es necesario realizar mediciones parámetros físicoquímicos adicionales y en diferentes épocas del año, de 
Nuevos reportes de humedales altoandinos. L. Vargas Porras

tal manera que se amplíe el conocimiento sobre los humedales de esta área del Chocó Biogeográfico.

\section{LITERATURA CITADA}

Allan, J. D. 1995. Stream ecology. Structure and function of running waters. London: Chapman \& Hall. 388 p.

Allan, J. D., A. J. Brenner, J. Erazo, L. Fernández, A. S. Flecker, D. L. Karwan, et al. 2002. Land use in watersheds of the Venezuelan Andes: A comparative analysis. Conserv Biol.16 (2): 527-38.

Carrizo R. 2008. Lineamientos y metodología a aplicar para la definición de "presupuestos mínimos» en materia de control de la contaminación hídrica. Buenoa Aires: Situación Ambiental de Argentina, PROGRAMA PRODIA. $30 \mathrm{p}$.

Castellanos, P. M., C. Serrato. 2008. Diversidad de macroinvertebrados acuáticos en un nacimiento de río en el páramo de Santurbán, Norte de Santander. Rev Acad Colomb Cienc. 32 (122): 79-86.

Cooke, R., J. Griggs, L. Sánchez, C. Díaz, D. Carvajal. 2001. Recopilación y presentación de datos de recursos ambientales y culturales en la región occidental de la cuenca del canal de Panamá. Volumen 4. Calidad Ambiental. Informe final de la región occidental de la cuenca del Canal. Panamá: Consorcio TLBG/UP/STRI.
Hernández Estela, F. C., D. Eusse, D. Artunduaga. 2006. Caracterización ambiental preliminar de los humedales de la meseta de Popayán y Puracé en el departamento del Cauca. Cali: CRC-WWF-CALIDRIS.

Fundación FEDENA, Corporación Autónoma del Valle del Cauca (CVC), Instituto Alexander Von Humboldt. 2004. Proyecto Páramo Andino, Páramo del Duende. Fenicia: Instituto Alexander Von Humboldt. 98 pp.

Gomi, T., R. C. Sidle, J. S. Richardson. 2002. Understanding processes and downstream linkages of headwater systems. BiosCience. 52 (10): $905-$ 16.

Goyenola, G. 2007. Guía del agua, conductividad. Versión 1.0. Montevideo: RED MAPSA. $10 \mathrm{p}$.

Ministerio de Agricultura. Decreto 1594 de 1984. Usos del agua y residuos líquidos. Bogotá: MinAgricultura.

Ministerio de Desarrollo Económico. 2000. Reglamento técnico del sector de agua potable y saneamiento básico RAS. Bogotá: MinDesarrollo.

Ministerio de Vivienda, Ordenamiento Territorial y Medio Ambiente de Uruguay. 2007. La calidad del agua del rio Uruguay, resultados de las tres primeras campañas. Montevideo: Dirección Nacional de Medio Ambiente.

Roldán, G. 1992. Fundamentos de limnología neotropical. Medellín: Universidad de Antioquia.

Segnini, S., M. Chacón. 2005. Caracterización fisicoquímica del hábitat interno y ribereño de ríos andinos en la cordillera de Mérida, Venezuela. Ecotropicos. 18 (1): 38-61. 